\title{
Design of the reliable collection and control scheme of the relay node status of the train carborne control system
}

\author{
Wan-Ting Zhao ${ }^{1, a}$, Gui-Ning Cao ${ }^{1}$, Xiang-Xian Chen ${ }^{1}$, Zhu-Jun Ling ${ }^{2}$, Zhen-Peng Nie ${ }^{2}$, Wen-Bin Tang ${ }^{2}$, Guo-Dong Teng ${ }^{2}$ \\ and Lan-Qing $\mathrm{Hu}^{2}$ \\ ${ }^{1}$ Department of Instrumentation Science and Engineering, Zhejiang University, Hangzhou, China \\ ${ }^{2}$ Research and Development Canter, Zhejiang United Science and Technology Co. Ltd, Hangzhou, China \\ awtzhao@zju.edu.cn
}

\begin{abstract}
Train operation control system is the core of urban rail transit system, and the train carborne control system is an important part of the whole train operation control systems, and its reliability will directly affect the safety of the train. In this paper, two out of three closed-loop dynamic input detection technology and hardware dynamic two out of two voting output technology and time synchronization technology were used to design relay node status acquisition and control scheme of the train carborne control system respectively, and effectively guaranteed the reliability of relay node status acquisition and control of the train carborne control system and therefore ensure the safety of operation of the train.
\end{abstract}

\section{Introduction}

The basic task of urban rail transit is transporting passengers in a safety, punctuality, high efficiency and high density way. In recent years, with the rapid development of Chinese economy and the acceleration of urbanization, urban rail transit has entered a period of rapid development1. With the continuous progress of science and technology, the rail transit signal control system has experienced the development process from manual control to automatic control. Automatic Train Control (ATC) System has been applied and gradually become the future development trend of signal control system. CBTC System is currently the most advanced implement of ATC system.

Train carborne control system is one of the core of urban rail transit system, which plays an important role in improving the operational efficiency of rail transit and ensuring the safety of train operation $[1,2]$.The train carborne control system is the core of the train operation control system, and also the guarantee of the normal operation of the transportation system [3].It is connected with some equipment or system such as the train, traction, line and rail. It also realizes the functions of operation controlling, train operation safety, automatic operation and scheduling management $[4,5]$.And therefore, the reliability of the system greatly affects the safety of the whole transport control system. [6]

The train carborne control system of relay node state acquisition and control scheme can effectively guarantee the reliability of the system to the data acquisition and control of the external device relay node working state, and effectively ensure safe operation of train carborne control system.

\section{Design of relay node state acquisition scheme}

The working state of external equipment relay node in train carborne control system is divided into two kinds of state, closed and separated. The correct collection of the state data is an important measure of knowing the real running condition and ensuring the running safety of trains. The status of the relay node of the train carborne control system is shown in Figure 1.

As can be seen from figure 1, the collection of the status of the relay node in train carborne control system uses two out of three closed-loop dynamic input detection schemes. The scheme consists of three parts, Input Output Vital (VIO) board, Signal Acquisition Conditional (SAC) board and CPU board. The VIO board input dynamic pulses through the channel $\mathrm{A}$, channel $\mathrm{B}$ and channel $\mathrm{C}$ respectively which are electrically separated from each other. And the dynamic pulse of the output format is 01010101, which controls the switch on each channel. When the external device nodes to relay is closed, three channels can collect dynamic pulses which are consistent with output pulses, when external equipment relay nodes is separated, three channels cannot get dynamic pulses which are consistent with output pulses. By the way, it can distinguish the two kinds of working states of the relay node for the external equipment effectively .Through three buses, the CAN1, 
CAN2 and CAN3, VIO board is connected with SAC1, SAC2 and SAC3 board respectively, which can achieve the transmission of the working status collection of the relay node in the external equipment by the channel $\mathrm{A}$, channel B and channel C. Three SAC boards are independent of each other, which are connected with CPU1, CPU2 and CPU3 through PCI1, PCI2 and PCI3 bus, and achieve the transmission of three channel state acquisition results of VIO board to three CPU boards. Through the point to point network communications, three CPU boards respectively exchange their respective received state acquisition results and take two to three votes. If the three CPU boards are collected the same dynamic pulse with three channel output pulses of VIO boards, the relay nodes of external equipment are considered to be closed, otherwise it is regarded that the relay nodes of external equipment are separated, which can realize the reliable acquisition to relay nodes of the train carborne control system.

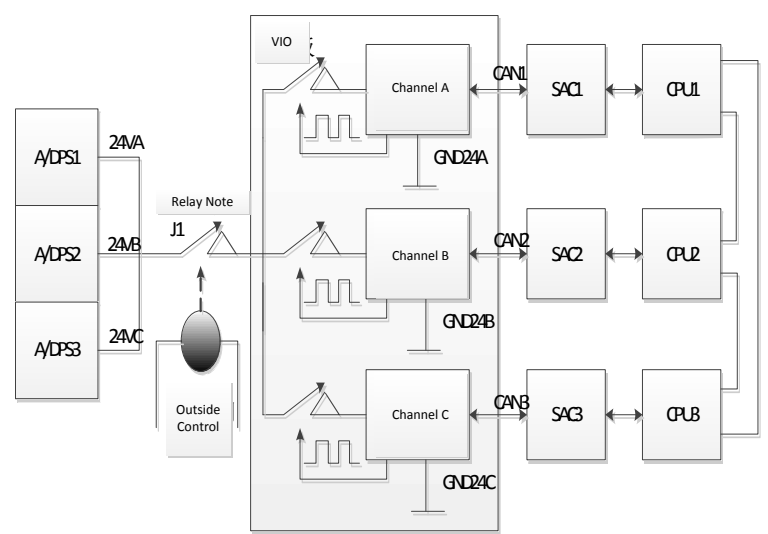

Fig.1 Scheme of Relay Node Status Acquisition

\section{Design of Relay Driving Scheme}

Reliable acquisition to the working state of the relay node is an effective guarantee to know about the train running state, and the reliable driving for relay nodes is an essential and important part of the correct control of train running state. The relay driver of the train carborne control system is shown in Figure 2.

As can be seen from Figure 2, the relay driver of the vehicle operation control system uses the hardware dynamic two take two voting output scheme. The output board is composed of channel $\mathrm{A}$ and channel $\mathrm{B}$, which is controlled by CPU1 and CPU2 of two processing units respectively. The ground circuit of external relay is control by relay SR which is independent. When the relay nodes are separated, the output board can output. When the relay nodes are closed, output board cannot output, by this way, it can realize the purpose of main standby switching by changing the operating condition of the relay.

When the system is working normally, the channel A and channel B will simultaneously output the dynamic pulse, and the voting circuit will be successful, therefore, the successful output will drive the external relay. When the system work abnormally, channels A and B cannot output dynamic pulse at the same time and voting circuit cannot output because of inconsistency, therefore, the failure of external relay drive would finally steer a safe system in this way, it can achieve the reliably drive of relay nodes of train carborne control system and ensure safe operation of the train.

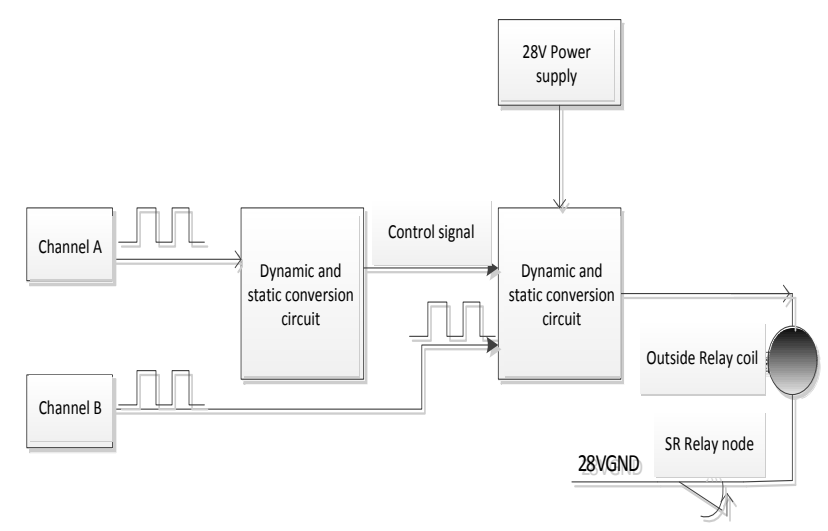

fig. 2 Relay Driving Scheme

\section{Design of CPU Time Synchronization}

In order to guarantee reliable data acquisition and control of working states of relay nodes in train carborne control system, we use the two out of three state acquisition schemes and two out of two relay driving scheme respectively. Both of these two schemes require reliable time synchronization between CPU.

In the time synchronization of the train carborne control system, a custom time synchronization protocol is adopted, which consists of four time stamps:

1) $\operatorname{org}($ Origin Timestamp) : Client time, The time that the client issues a time synchronization request;

2) rec (Timestamp Receive): Server time, The time that the server receives the client time synchronization request;

3) xmt (Timestamp Transmit):Server time, The time that the server sends a time synchronization response;

4) dst (Destination Timestamp):Client time, The time that the client receives the server time synchronization response;

5) The process of completing the multiple CPU time synchronization of the train carborne control system is shown in Figure 3.

As can be seen from Figure 3, the time synchronization is divided into five steps, respectively as follows:

1. At time $\mathrm{T} 1$, the client sends a time synchronization request that contains the $\mathrm{t} 1$ value to server, and copies the t1 value to the local state variable $\mathrm{T} 1$;

2. At time $\mathrm{T} 2$,the server receives the time synchronization request which is sent by the client, and the t2 value and the org time stamps in time synchronization request were copied to the local state variables $\mathrm{T} 2$ and $\mathrm{T} 1$ respectively; 
3. At time $\mathrm{T} 3$, the server sends the rec time stamp which contains a $\mathrm{t} 2$ value and time synchronization response of xmt time stamp which contains a $\mathrm{t} 3$ value to the client, and copy the $\mathrm{t} 3$ value to the local state variable T3;

4. At timeT4, the client receives the time synchronization response which is sent by the service, and the $\mathrm{t} 4$ value and the rec time stamp in time synchronization response, xmt time stamp were copied to the local state variables T4 and T2, T3 respectively;

5. After judging the legality of all data, by using formula (1) and formula (2), the client can calculate the time warp offset and round trip delay of the server with respect to the client, thus completing multiple CPU time synchronization of the vehicle operation control system.

Real offset should be in the range shown in the formula (3) because of the system time precision .The lambda in formula (3) is the synchronous distance, the value of which is shown in formula (4) The epsilon in the formula (4) is the dispersion, and its value varies with time, which is shown in the formula (5): The r.rho, s.rho and PHI In formula (6) respectively express time precision of the data packet, time precision of the system and the system frequency tolerance.

By using the above formulas, the range of the time variation offset of the server and the client can be calculated, and a plurality of different ranges of values can be obtained after the time synchronization. By using these different ranges of values, the interval of offset value which contains the highest confidence can be found through Marzullo's Algorithm. Therefore, it can guarantee the time synchronization between the multiple CPU mostly, and provide the guarantee for the reliable collection and control of the relay node status of the vehicle operation control system.

\begin{tabular}{|c|c|c|c|c|}
\hline server & & t3 & t6 & t7 \\
\hline & / & $\begin{array}{l}\vdots \\
\vdots \\
\backslash\end{array}$ & $\begin{array}{l}\text { / } \\
\text { / }\end{array}$ & $\begin{array}{l}\vdots \\
\vdots \\
\backslash\end{array}$ \\
\hline client & & t4 & t5 & t8 \\
\hline
\end{tabular}

Fig. 3 Time Synchronization Process of Train Operation Control System

\section{Summary}

In this paper, two out of three closed-loop dynamic input detection technologies and hardware dynamic two out of two voting output technology were used to design relay node status acquisition and control scheme of the train carborne control system respectively. It can effectively guarantee the reliable collection and control of the working status of each relay node. And it also effectively ensures the reliability and safety of train carborne control system, and prevents the train from abnormal operation because of status acquisition or control error of the relay node in the train control system, which can reduce the occurrence of railway traffic accidents.

\section{Acknowledgment}

This work is supported by the funding from the National Science and Technology Infrastructure Program of China under Grant 2015BAG19B03

\section{References}

1. Duan Hongwei. Research on Distributed Vehicle Operation Control System for Urban Mass Transit[D]. Beijing Jiaotong University, 2013.

2. Yan Fei. The Formal Modelling and Model Checking Research of Rail Transportation Train Control System[D]. Beijing Jiaotong University, 2006.

3. Zhang Yadong. Study on the Safety Risk Identification and Analysis of Train Control System of High-speed Railway[D]. Southwest Jiaotong University, 2013.

4. Song Yan. Design and Implementation of the Principle Prototype for Tuberail On-board Operation Control System[D]. Beijing Jiaotong University, 2009.

5. Yuan Zhibin. Development of Novel Vehicle Operation Control System Elementary Prototype for Modern Tram[D]. Beijing Jiaotong University, 2012.

6. Panfeng Liu, Xixi Qiu, Xiangxian Chen. Urban Rail Transit Train Automatic Protection Operation System Software Design[J].Computer Engineering.2012,38(19): 1-5

7. Rail Transit Vehicle Interface Standards Committee of the IEEE Vehicular Society.IEEE Standard for Communications - Based Train Control(CBTC ) Performanceand Functional Requirements[M].New York :IEEE Publisher, 1999 :5-8 